\title{
Características Tecnológicas de la Fibra Blanca de Alpaca Huacaya en la Zona Altoandina de Apurímac
}

\author{
Technological Characteristics of the White Fibre of Huacaya Alpaca \\ in the Andean Region of ApUrimac
}

Rutnis Vásquez O. ${ }^{1}$, Oscar Elisban Gómez-Quispe ${ }^{1,2}$, Edgar Quispe P. ${ }^{1}$

\section{Resumen}

\begin{abstract}
Con el objeto de estimar cinco características tecnológicas de la fibra de alpaca Huacaya color blanco en una comunida, de la zona altoandina de Apurímac, Perú, se evaluaron 405 muestras tomadas de la zona media del costillar, antes de la esquila. Las variables estudiadas fueron: media del diámetro de fibra (MDF), coeficiente de variación del diámetro de la fibra (CVDF), índice de confort (IC), índice de curvatura (ICur) y finura al hilado (FiHi), según sexo y grupo etario de acuerdo a la dentadura (DL=Dientes de leche, $2 \mathrm{D}=2$ dientes, $4 \mathrm{D}=4$ dientes, $\mathrm{BLL}=$ boca llena). La MDF para machos y hembras fue de 19.6 y $20.1 \mu \mathrm{m}$, respectivamente $(\mathrm{p}<0.05)$, donde los valores aumentaron con la edad $(\mathrm{p}<0.05)$. No hubo diferencias en el CVDF por sexos ni entre grupos etarios. El IC fue de $96.8 \%$ en machos y $95.5 \%$ en hembras $(\mathrm{p}<0.05)$, disminuyendo conforme aumenta la edad $(p<0.05)$. El ICur fue similar entre sexos y la diferencia entre edades solo se aprecia entre DL con 4D y BLL $(p<0.05)$. La FiHi fue diferente entre sexos $(p<0.05)$ y entre los grupos etarios DL y BLL $(p<0.05)$. Se encontraron relaciones significativas positivas entre MDF con FiHi e IC con ICur y negativas entre MDF con IC, MDF con ICur, IC con FiHi, ICur con FiHi e ICur con CVDF. Los resultados indican que en esta zona del país existen alpacas con buen potencial en calidad de fibra.
\end{abstract}

Palabras clave: camélidos, textiles, Andes, finura

\section{Abstract}

The study aimed to estimate five technological characteristics of the white fibre of Huacaya alpaca of a rural community of the Andean region of Apurimac, Peru. Fibre samples $(n=405)$ were taken from the middle of the rib area prior to shearing. The variables studied were: mean diameter of fibre (MDF), coefficient of variation of fibre diameter

\footnotetext{
${ }^{1}$ Facultad de Medicina Veterinaria y Zootecnia, Universidad Nacional Micaela Bastidas de Apurímac, Perú

${ }^{2}$ E-mail: oegomezq@hotmail.com
}

Recibido: 12 de junio de 2014

Aceptado para publicación: 15 de enero de 2015 
(CVDF), comfort index (IC), curvature index (ICur) and spinning fineness (FiHi), by sex and age group according to teeth $(\mathrm{DL}=$ Deciduous teeth, $2 \mathrm{D}=2$ permanent teeth, $4 \mathrm{D}=4$ teeth, BLL=all teeth). The MDF for males and females was 19.6 and $20.1 \mu \mathrm{m}$ respectively $(p<0.05)$, where values increased with age $(p<0.05)$. No statistical difference was found by sex or age for CVDF. The IC was $96.8 \%$ in males and $95.5 \%$ in females $(p<0.05)$, decreasing with age $(\mathrm{p}<0.05)$. The ICur was similar between sexes and difference due to age was only observed between DL with $4 \mathrm{D}$ and BLL $(\mathrm{p}<0.05)$. The FiHi was different between sexes $(p<0.05)$ and between Dl and BLL $(p<0.05)$. Significant and positive relationships was found between MDF with FiHi and IC with ICur, and negatives between MDF with IC, MDF with ICur, IC with FiHi, ICur with FiHi, and ICur with CVDF. These results indicate that in this area of the country there are alpacas with good potential in fibre quality.

Key words: camelids, textiles, Andes, finesse

\section{INTRODUCCIÓN}

Las zonas altoandinas de los Andes peruanos albergan una considerable cantidad de habitantes, cuya principal fuente económica de recurso renovable está basada en la crianza de camélidos sudamericanos. La región de Apurímac ocupa el quinto lugar en producción alpaquera, con cerca de 225000 cabezas de las más de 3590000 que se cuentan a nivel nacional (INEI, 2012); sin embargo, el aprovechamiento de la fibra de este animal es limitado en la zona, pese a que se dispone de un gran potencial de exportación (GRA, 2006). Es así que esta actividad se constituye en una estrategia de lucha contra la pobreza que afecta a las comunidades campesinas alpaqueras.

La finura de la fibra está directamente relacionada con la media del diámetro de fibra (MDF). La clasificación de los vellones se basa principalmente en esta característica, ya que permite una mejor valoración al momento de la comercialización; es decir, vellones de mejor calidad tienen mejores precios (Quispe, 2010).

Existen, además, otras características complementarias que determinan la calidad de la fibra (Quispe, 2010). Entre ellas se tiene el coeficiente de variación del diámetro de la fibra (CVDF), que es una medida de amplitud relativa del diámetro de la fibra alrededor de la media dentro de un vellón, de manera que un vellón con CVDF más bajo indica una mayor uniformidad de los diámetros de las fibras individuales que lo componen, produciendo un hilo más resistente (Manso, 2011). Otra característica es el índice de confort (IC) o factor de comodidad, entendiéndose que a mayor confort se tiene fibras de menor diámetro (Quispe et al., 2013). El índice de curvatura (ICur) de la fibra es una característica textil adicional que se utiliza para describir la propiedad espacial de una masa de fibras (Fish et al., 1999). La finura al hilado (FiHi) es un estimador del rendimiento de la muestra cuando es hilado y convertido en hilo (Manso, 2011). En este contexto, las correlaciones entre estas variables permiten conocer la manera como están relacionadas entre ellas.

En Apurímac existe poca información sobre la calidad de la fibra de las alpacas criadas en la zona, de allí que el presente trabajo tuvo como objetivo determinar las características tecnológicas de la fibra de alpaca Huacaya color blanco, con respecto a MDF, CVDF, IC, ICur y FiHi, según sexo y grupo etario, así como estimar las relaciones entre estas variables para poder trazar una línea de base en el conocimiento del grado de avance de la calidad de fibra de alpaca. Esto, en 
su momento, permitirá establecer las bases para la implementación de programas de mejoramiento genético que permitan mejorar la productividad de los rebaños y elevar el nivel de vida del productor andino.

\section{Materiales y Métodos}

El estudio se realizó durante el primer semestre de 2012 en la comunidad de Iscahuaca, distrito de Cotaruse, provincia de Aymaraes, ubicada en la región de Apurímac. Es una zona de Puna Seca entre 3700 y 5300 msnm, con temperaturas de -9 a $-5{ }^{\circ} \mathrm{C}$ por las noches y 10 a $25{ }^{\circ} \mathrm{C}$ en el día, con una precipitación pluvial de $700 \mathrm{~mm} / \mathrm{año}$.

Se tomaron $20 \mathrm{~g}$ de muestra de lana de 405 alpacas antes de la esquila y con el animal en pie. Las muestras se tomaron del costillar medio (Aylan-Parker y McGregor, 2002) y se colocaron en bolsas de polietileno, con la identificación del sexo y edad de los animales.

El análisis de las características físicas (MDF, CVDF, IC, ICur, FiHi) se realizó en el laboratorio de Lanas y Fibras de la Universidad Nacional de Huancavelica, utilizando el equipo OFDA 2000 (Australia), según lo propuesto por Brims et al. (1999).

Las cinco variables se evaluaron a través de un análisis de varianza según un diseño completamente al azar con arreglo factorial de $2 \times 4$, tomando en cuenta el factor sexo en dos niveles ( $\mathrm{M}=$ machos; $\mathrm{H}=$ hembras) y el factor estrato etario en cuatro niveles de acuerdo a la dentadura $(\mathrm{DL}=$ dientes de leche; $2 \mathrm{D}=2$ dientes; $4 \mathrm{D}=4$ dientes; BLL = boca llena).

Para la comparación de medias se utilizó la prueba de Duncan $(\alpha=0.05)$. Previamente se evaluó el cumplimiento de los su- puestos de normalidad, homogeneidad de varianzas e independencia de errores. Solo se requirió la transformación logarítmica para el índice de confort (IC). Asimismo, para evaluar las relaciones entre las características físicas de la fibra de alpaca se utilizó la correlación de Pearson.

El procesamiento y análisis de datos se realizó utilizando el software estadístico R3.1.2 for Windows.

\section{Resultados}

Las principales características físicas encontradas en la fibra de alpaca Huacaya de color blanco se observan en el Cuadro 1 . El diámetro de la fibra es menor en machos que en hembras $(\mathrm{p}<0.05)$, y estos valores aumentan conforme aumenta la edad en los grupos etarios $(\mathrm{p}<0.05)$; sin embargo, la variabilidad del diámetro de la fibra es similar tanto entre sexos como entre grupos etarios.

El índice de confort es diferente entre sexos y entre grupos etarios, disminuyendo conforme aumenta la edad $(\mathrm{p}<0.05)$. El índice de curvatura es similar entre sexos y la diferencia entre edades solo se aprecia entre DL con 4D y BLL. Por otro lado, la finura al hilado es diferente entre sexos $(p<0.05)$ y entre los grupos etarios DL y BLL $(p<0.05)$.

Asimismo, existen relaciones positivas entre MDF con FiHi, IC con ICur, y FiHi con CVDF; así como relaciones negativas entre MDF con IC, MDF con ICur, IC con FiHi, ICur con FiHi e ICur con CVDF (Cuadro 2).

La Fig. 1 muestra la relación entre la clasificación oficial de la fibra - Norma Técnica Peruana (N.T.P.) 231.301.2014 (INDECOPI, 2014) y los resultados de MDF obtenidos. 
Cuadro 1. Medias \pm error estándar del diámetro de fibra (MDF), coeficiente de variación del diámetro de fibra (CVDF), índice de confort (IC), índice de curvatura (ICur) y finura al hilado (FiHi), según sexo y edad de alpacas Huacaya en la comunidad de Iscahuaca (Cotaruse, Apurímac)

\begin{tabular}{ccccccc}
\hline Factor $^{1}$ & $\mathrm{n}$ & $\begin{array}{c}\text { MDF } \\
(\mu \mathrm{m})\end{array}$ & $\begin{array}{c}\text { CVDF } \\
(\%)\end{array}$ & $\begin{array}{c}\text { IC } \\
(\%)\end{array}$ & $\begin{array}{c}\text { ICur } \\
(\% \mathrm{~mm})\end{array}$ & $\begin{array}{c}\text { FiHi } \\
(\mu \mathrm{m})\end{array}$ \\
\hline $\begin{array}{c}\text { Sexo } \\
\text { M }\end{array}$ & 158 & $19.6 \pm 0.2^{\mathrm{a}}$ & $21.2 \pm 0.2^{\mathrm{a}}$ & $96.8 \pm 0.3^{\mathrm{a}}$ & $36.9 \pm 0.5^{\mathrm{a}}$ & $19.1 \pm 0.2^{\mathrm{a}}$ \\
H & 247 & $20.1 \pm 0.2^{\mathrm{b}}$ & $21.3 \pm 0.1^{\mathrm{a}}$ & $95.5 \pm 0.4^{\mathrm{b}}$ & $37.1 \pm 0.4^{\mathrm{a}}$ & $19.6 \pm 0.2^{\mathrm{b}}$ \\
Edad & & & & & & \\
DL & 129 & $17.8 \pm 0.2^{\mathrm{a}}$ & $21.3 \pm 0.2^{\mathrm{a}}$ & $98.7 \pm 0.2^{\mathrm{a}}$ & $35.8 \pm 0.5^{\mathrm{a}}$ & $17.4 \pm 0.2^{\mathrm{a}}$ \\
2D & 81 & $19.7 \pm 0.3^{\mathrm{b}}$ & $21.2 \pm 0.2^{\mathrm{a}}$ & $97.2 \pm 0.4^{\mathrm{b}}$ & $36.9 \pm 0.8^{\mathrm{ab}}$ & $19.2 \pm 0.2^{\mathrm{ab}}$ \\
4D & 98 & $20.7 \pm 0.3^{\mathrm{c}}$ & $21.1 \pm 0.2^{\mathrm{a}}$ & $95.2 \pm 0.7^{\mathrm{c}}$ & $37.6 \pm 0.7^{\mathrm{b}}$ & $20.2 \pm 0.3^{\mathrm{ab}}$ \\
BLL & 97 & $22.1 \pm 0.3^{\mathrm{d}}$ & $21.3 \pm 0.2^{\mathrm{a}}$ & $92.3 \pm 0.8^{\mathrm{d}}$ & $38.2 \pm 0.7^{\mathrm{b}}$ & $21.6 \pm 0.3^{\mathrm{b}}$ \\
\hline Total & 405 & $19.9 \pm 0.2^{2}$ & $21.2 \pm 0.1^{1}$ & $96.0 \pm 0.3$ & $37.0 \pm 0.3$ & $19.4 \pm 0.2^{2}$ \\
\hline
\end{tabular}

a,b,c,d Medias con superíndices diferentes dentro de columnas y factor denotan diferencia estadística $(p<0.05)$

${ }^{1}$ Sexo: $\mathrm{M}=$ machos, $\mathrm{H}=$ hembras; $\mathrm{Edad}$ : $\mathrm{DL}=$ diente de leche, $2 \mathrm{D}=2$ dientes, $4 \mathrm{D}=4$ dientes, $\mathrm{BLL}=$ boca llena

Cuadro 2. Correlaciones de Pearson entre las características físicas de la fibra de alpaca Huacaya en la comunidad de Iscahuaca (Cotaruse, Apurímac)

\begin{tabular}{lcccc}
\hline Variables $^{1}$ & MDF & IC & Icur & FiHi \\
\hline \multirow{2}{*}{ IC } & -0.74891 & & & \\
& $<.0001$ & & & \\
Icur & -0.46076 & 0.39484 & & \\
& $<.0001$ & $<.0001$ & & \\
FiHi & 0.99182 & -0.75457 & -0.47802 & \\
& $<.0001$ & $<.0001$ & $<.0001$ & \\
CVDF & -0.02818 & -0.07941 & -0.15921 & 0.08049 \\
& 0.5717 & 0.1106 & 0.0013 & 0.1058 \\
\hline
\end{tabular}

${ }^{1} \mathrm{MDF}=$ Media del diámetro de fibra, IC= Índice de confort, ICur = Índice de curvatura, FiHi= Finura al hilado, CVDF = Coeficiente de variación del diámetro de fibra 


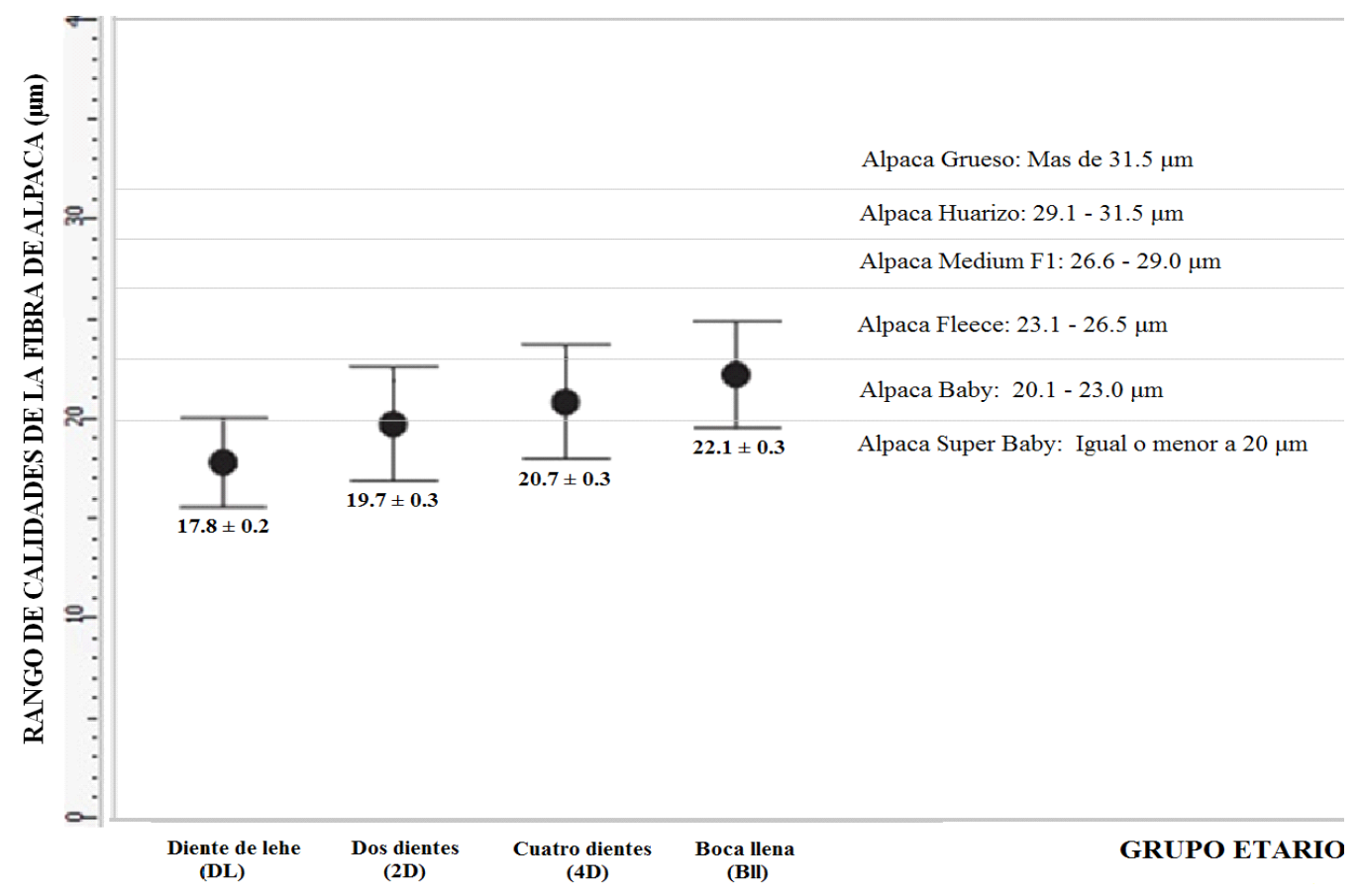

Figura 1. Clasificación de la fibra por calidades (según Norma Técnica Peruana de la fibra de alpaca, N.T.P. 231.301.2014) y la media del diámetro de fibra (MDF) \pm error estándar por grupo etario en la comunidad de Iscahuaca (Cotaruse, Apurímac)

\section{Discusión}

\section{Características Tecnológicas de la Fibra}

\section{Media del diámetro de fibra}

En general, la MDF encontrada fue de $19.9 \mu \mathrm{m}$, siendo más fina o superior a los reportados por otros autores (Ponzoni, 2000; Aylan-Parker y McGregor, 2002; McGregor y Butler, 2004; Quispe et al., 2007, 2013; Montes et al., 2008; Quispe, 2010). Los resultados de MDF conllevan a considerar que un alto porcentaje de estas fibras son de buena calidad, de acuerdo a la actual clasificación de la Norma Técnica Peruana (N.T.P.) 231.301.2014 (INDECOPI, 2014). Esto indicaría la presencia de animales con buena calidad de fibra, en especial de machos, lo que facilitaría la selección de una élite de reproductores en las comunidades alpaqueras.
Una de las causas más probables de esta finura podría deberse a la labor realizada por los productores altoandinos en la selección de animales machos, así como a la introducción de reproductores provenientes de Puno y Cusco, que fue realizado con el apoyo de instituciones públicas y privadas. También, podría deberse a que las fibras de los animales deficientemente alimentados son más finas pero menos resistentes que las fibras de los animales con mejor alimentación; problema que ocurre durante los periodos de sequía constante en estas comunidades (Contreras, 2009).

La mayor finura del diámetro de la fibra (MDF) en los machos (19.6 $\mu \mathrm{m})$, en comparación con las hembras $(20.1 \mu \mathrm{m})$, resulta contradictoria con el reporte de Lupton et al. (2006) y Aylan-Parker y McGregor (2002), quienes indican que los machos tienen mayor diámetro de fibra. También difiere al encon- 
trado por Wuliji et al. (2000) y McGregor y Butler (2004), quienes informan que no existe efecto del sexo sobre la MDF; sin embargo, concuerda con lo encontrado por Montes et al. (2008) y Quispe et al. (2009a). Es posible que los resultados del presente estudio se deban a la selección subjetiva de los machos, la cual es mucho más minuciosa que la selección de las hembras (Quispe et al., 2009a), y a que las hembras son generalmente de la misma región de Apurímac, mientras que los machos provienen de otras regiones.

La MDF incrementó significativamente $(\mathrm{p}<0.05)$ según grupo etario o conforme avanza la edad del animal (Cuadro 1), tal como se señala en la literatura (Wuliji et al., 2000; McGregor y Butler, 2004; Quispe et al., 2009b). Sin embargo, las medias obtenidas en el presente estudio fueron más finas o superiores a los valores reportados por Huamaní y Gonzáles (2004), Lupton et al. (2006), Mamani (2006), Carrasco (2009) y Encinas (2009) según los estratos etarios. El efecto de la edad sobre la MDF se debería a la queratinización de la fibra, que resulta en un mayor proceso de modulación en animales adultos (Contreras, 2009). Además, Rogers (2006) indica que las alpacas adultas producen vellones cada vez menos finas debido al efecto de las esquilas, que tiende a incrementar el funcionamiento folicular.

Diversos autores señalan, asimismo, que la edad influye sobre casi todas las características físicas de la fibra de alpacas (Bustinza, 2001; Lupton et al., 2006; McGregor, 2006; Quispe et al., 2007; Valdivia, 2009). Asimismo, Bustinza (2001) considera que existen factores adicionales, entre ellos, la nutrición, que juegan un rol importante en la formación, maduración folicular, crecimiento y diámetro de la fibra.

Coeficiente de variación del diámetro de fibra

El coeficiente de variación del diámetro de fibra (CVDF) no fue afectado por el sexo de las alpacas, lo cual concuerda con lo reportado por McGregor y Butler (2004) y Quispe et al. (2009b). Al respecto, se sabe que el CVDF tiene alta influencia sobre algunas propiedades requeridas en la industria textil (Lupton et al., 2006), pues conjuntamente con el MDF determinan la finura al hilado de la fibra que está asociado al rendimiento del hilado (McGregor, 2006). Además, tiene un efecto sobre la resistencia a la tracción, pues fibras con mayor CVDF tienen menor resistencia (Mueller, 2000), afectando el rendimiento al cardado, al peinado y del tejido durante el proceso de transformación textil de la fibra (Wang et al., 2003).

Del mismo modo, el CVDF no estuvo influenciado por el estrato etario, tal y como lo menciona Quispe et al. (2009a) en la región de Huancavelica, Perú, aunque con menor variabilidad. Asimismo, los valores son inferiores a otros reportes (Aylan-Parker y McGregor, 2002; Wang et al., 2003; McGregor y Butler, 2004; Lupton et al., 2006), donde se mencionan valores de CVDF entre 23.5 y $28.1 \%$. Solo McGregor y Butler (2004) encontraron evidencia de que el CVDF disminuye rápidamente hasta los 2 a 3 años, para luego incrementar levemente hasta los 10 años de edad.

\section{Índice de confort}

Los índices de confort (IC) por sexo fueron de 96.8 y $95.5 \%$ en machos y hembras, respectivamente $(\mathrm{p}<0.05)$, los cuales corresponden a factores de picazón (FP) o fibras $\geq 30 \mu \mathrm{m}$ de 3.2 y $4.5 \%$ en machos $\mathrm{y}$ hembras, respectivamente. El FP implica que si los extremos de las fibras que sobresalen de la superficie de los hilos fueran delgados, estas serían más flexibles y menos probable que provoquen picazón en la piel. El IC del presente estudio fue superior a otros reportes (McGregor y Butler, 2004; Lupton et al., 2006; Quispe et al., 2007, 2013). Según Quispe et al. (2013), los consumidores sienten picazón con textiles que contienen más del $5 \%$ de fibras mayores de $30 \mu \mathrm{m}$. 
El IC por estrato etario fue estadísticamente significativo, siendo menor conforme avanza la edad $(\mathrm{p}<0.05)$. Sin embargo, es necesario realizar una corrección por efectos medioambientales, pues el factor nutrición podría estar afectando el IC (Quispe et al., 2007).

Índice de curvatura

Según Holt (2006), una curvatura menor de 50 grados $/ \mathrm{mm}$ se describe como curvatura baja; de allí que el índice de curvatura (ICur) estimado a nivel general (37.0 grados/ $\mathrm{mm}$ ) en el presente estudio correspondería a una fibra con baja cantidad de rizos. Estos valores de ICur fueron inferiores a los encontrados por Siguayro (2009). Sin embargo, otros autores reportan valores más bajos, entre 28.0 y 32.2 grados $/ \mathrm{mm}$ (Liu et al., 2004; Wang et al., 2004; Lupton et al., 2006; McGregor, 2006). Por otro lado, Holt (2006) encuentra valores con un rango más amplio (25 a 60 grados $/ \mathrm{mm}$ ).

En el presente trabajo no se encontró efecto del sexo sobre el ICur. Diferencias significativas por estrato etario solo se aprecian entre DL con 4D y BLL (Cuadro 1). Al respecto, McGregor (2006) menciona que la edad no afecta el ICur en alpacas Huacaya, pero encuentra diferencias en alpacas Suri.

\section{Finura al hilado}

La finura al hilado (FiHi) es un estimador del rendimiento de la muestra cuando es hilado y convertido en hilo (Manso, 2011).

El valor de la FiHi (19.1 en machos y $19.6 \mu \mathrm{m}$ en hembras) se aproxima al valor de $20.9 \mu \mathrm{m}$ obtenido en alpacas Huacaya por Quispe (2010). La FiHi fue diferente entre los grupos etarios DL y BLL $(\mathrm{p}<0.05)$. Diversos reportes indican, por otro lado, que la $\mathrm{HiFi}$, al igual que otras características físicas de la fibra, se incrementan con la edad (Bustinza, 2001; Lupton et al., 2006; McGregor, 2006; Quispe et al., 2007; Valdivia, 2009) y podría estar influida por factores nutricionales (Bustinza, 2001).

\section{Relaciones entre las Características Tec- nológicas}

\section{Relaciones entre MDF e ICur}

Se encontró una relación negativa $(-0.46)$ y moderada entre MDF e ICur, relativamente similar a los resultados de McGregor (2006) quien reportó una relación negativa (-0.16) en el sur de Australia. Asimismo, Lupton et al. (2006) informaron de una relación negativa alta (-0.86) en EEUU, y Safley (2005) de -0.72 , donde fibras con alta curvatura tenían menor diámetro.

\section{Relación entre $M D F$ y CVDF}

La relación de -0.028 entre MDF y CVDF no fue significativa. Esta relación negativa y muy baja fue similar a los reportes de Quispe et al. (2009b), quienes reportaron una relación de - 0.088 en Huancavelica, Perú; aunque en Australia, Ponzoni et al. (1999) informaron una relación de 0.01 entre estas variables.

\section{Relación entre CVDF e IC}

Respecto a las variables CVDF e IC en la fibra de alpaca Huacaya color blanco, no se encontró una relación significativa entre ambas $(-0.079 ; \mathrm{p}=0.1106)$. La información respecto a esta relación es muy limitada.

\section{Relación entre MDF e IC}

La relación negativa y alta encontrada entre MDF e IC (-0.75) estuvo muy cercana a lo reportado por Cervantes et al. (2010) de -0.968, y concuerda con resultados de otros reportes (Ponzoni et al., 1999; Lupton et al., 2006; Quispe et al., 2007; Contreras, 2009).

\section{Relación entre CVDF e ICur}

La relación entre CVDF e ICur (-0.16) fue negativa y muy baja, próxima al valor de -0.08 reportado por McGregor (2006) en el sur de Australia. 


\section{Conclusiones}

Se observan indicadores favorables en las características físicas, así como relaciones positivas y negativas entre estas variables de la fibra blanca de alpaca Huacaya en la comunidad de Iscahuaca (Cotaruse, Apurímac, Perú). Estos resultados permiten trazar una línea de base en la zona para el seguimiento del grado de avance de la calidad de la fibra de alpaca.

\section{Agradecimientos}

Los autores agradecen a la Universidad Nacional Micaela Bastidas de Apurímac por el financiamiento del estudio, al Programa de Mejora de Camélidos Sudamericanos (PROCASUD) de la Universidad Nacional de Huancavelica por el apoyo en el análisis de las muestras y a los directivos de la comunidad campesina de Iscahuaca (Cotaruse, Aymaraes, Apurímac), por su apoyo en las coordinaciones con los criadores alpaqueros para la obtención de las muestras.

\section{Literatura Citada}

1. Aylan-Parker J, McGregor BA. 2002. Optimising sampling techniques and estimating sampling variance of fleece quality attributes in alpacas. Small Rumin Res 44: 53-64. doi: 10.1016/S09214488(02)00038-X

2. Brims MA, Peterson AD, Gherard SG. 1999. Introducing the OFDA 2000 for rapid measurement of diameter profile on greasy wool staples. International Wool Textile Organization. Western Australia: Report No RWG 04.

3. Bustinza V. 2001. La alpaca, conocimiento del gran potencial andino. Puno: Ed Universidad Nacional del Altiplano. $496 \mathrm{p}$.

4. Carrasco J. 2009. Proporción de pelos en vellones clasificados según edad en alpaca Huacaya hembra. Tesis de Mé- dico Veterinario y Zootecnista. Puno: Univ Nacional del Altiplano. 56 p.

5. Cervantes I, Pérez-Cabal MA, Morante R, Burgos A, Salgado C, Nieto B, Goyache F, et al. 2010. Genetic parameters and relationships between fibre and type traits in two breeds of Peruvian alpacas. Small Rumin Res 88 : 6-11. doi: 10.1016/j.smallrumres. 2009.10.016

6. Contreras A. 2009. Estructura cuticular y características físicas de la fibra de alpaca Huacaya (Vicugna pacos) de color blanco en la Región de Huancavelica. Tesis de Ingeniero Zootecnista. Huancavelica: Univ Nacional de Huancavelica. 90 p.

7. Encinas M. 2009. Caracterización de la fibra de alpacas Huacaya del Instituto de Investigación y Promoción de Camélidos Sudamericanos de la Universidad Nacional del Altiplano. Tesis de Médico Veterinario y Zootecnista. Puno: Univ Nacional del Altiplano. 59 p.

8. Fish VE, Mahar TJ, Crook BJ. 1999. Fibre curvature morphometry and measurement. Wool Tech Sheep Breed 48:248-265.

9. [GRA] Gobierno Regional de Apurímac. 2006. Plan estratégico regional de Apurímac (PERX). Abancay: Oficina de Planificación del Gobierno Regional de Apurímac.

10. Holt C. 2006. A survey of the relationships of crimp frequency, micron, character and fibre curvature. A report to the Australian alpaca association. [Internet]. Disponible en: http://www. cameronholt.com/CrimpRelationships.pdf

11. Huamaní R, Gonzáles CE. 2004. Efecto de la edad y el sexo en los parámetros físicos de la fibra de alpaca (Lama pacos) Huacaya en Huancavelica. Tesis de Ingeniero Zootecnista. Huancavelica: Univ Nacional de Huancavelica. $80 \mathrm{p}$.

12. [INDECOPI] Instituto Nacional de Defensa del Consumidor y Propiedad Intelectual. 2014. Norma Técnica Pe- 
ruana de la fibra de alpaca. Perú: N.T.P. 231.301.2014.

13. [INEI] Instituto Nacional de Estadística e Informática. 2012. IV Censo Nacional Agropecuario 2012 (CENAGRO 2012). Lima: Resultados Preliminares. $91 \mathrm{p}$.

14. Liu X, Wang L, Wang X. 2004. Evaluating the softness of animal fibers. Text Res J 74: 535-538.

15. Lupton CJ, McColl A, Stobart RH. 2006. Fiber characteristics of the Huacaya alpaca. Small Rumin Res 64: 211-224. doi: 10.1016/j.smallrumres.2005.04.023

16. Mamani A. 2006. Correlación entre el diámetro, densidad y rizo de la fibra de alpaca Huacaya hembra, según región corporal. Tesis de Médico Veterinario y Zootecnista. Puno: Univ Nacional del Altiplano. $56 \mathrm{p}$.

17. Manso C. 2011. Determinación de la calidad de fibra de alpaca en Huancavelica (Perú): Validación de los métodos de muestreo y valoración. Tesis de Ingeniero Agrónomo. Pamplona: Univ Pública de Navarra. $121 \mathrm{p}$.

18. McGregor BA, Butler KL. 2004. Sources of variation in fibre diameter attributes of Australian alpacas and implications for fleece evaluation and animal selection. Aust J Agric Res 55: 433-442. doi:10.1071/AR03073

19. McGregor BA. 2006. Production attributes and relative value of alpaca fleeces in southern Australia and implications for industry development. Small Ruminant Research 61: 93-111. doi: 10.1016/j.smallrumres.2005.07.001

20. Montes M, Quicaño I, Quispe E, Alfonso L. 2008. Quality characteristics of Huacaya alpaca fibre produced in the Peruvian Andean Plateau region of Huancavelica. Span J Agric Res 6(1): 33-38. doi: 10.5424/sjar/2008061-5258

21. Mueller J. 2000. Mejoramiento genético de la lana. Caracteres de importancia. Instituto Nacional de Tecnología Agropecuaria, EEA Bariloche. En: III Congreso Lanero, Trelew, 2000. Dis- ponible en: http://www.minagri.gob.ar/ site/ganaderia/ovinos/04=Documen taci \% C3\%B3n\%20 Tecnica/ 04 Genetica/_archivos/000000_Mejoramiento $\% 2$ ogen $\%$ C3\% A9tico $\% 20 \mathrm{de} \%$ 20la\%20lana.pdf

22. Ponzoni RW, Grimson RJ, Hill JA, Hubbard DJ, McGregor BA, Howse $A$, et al. 1999. The inheritance of and association among some production traits in young Australian alpacas. Aust Adv Anim Breed Gen 13: 468-471.

23. Ponzoni $R \boldsymbol{W}$. 2000. Genetic improvement of Australian alpacas: present state and potential developments. Proc Aust Alpaca Assoc 1: 71-96.

24. Quispe E, Flores A, Alfonso L, Galindo A. 2007. Algunos aspectos de la fibra y peso vivo de alpacas Huacaya de color blanco en la región de Huancavelica. En: XX Reunión ALPA. Cusco: Asociación Latinoamericana de Producción Animal.

25. Quispe EC, Alfonso L, Flores A, Guillén H, Ramos Y. 2009a. Bases para un programa de mejora de alpaca en la región altoandina de HuancavelicaPerú. Arch Zootec 58: 705-716.

26. Quispe EC, Rodríguez T, Iñiguez L, Mueller J. 2009b. Producción de fibra de alpaca, llama, vicuña y guanaco en Sudamérica. Anim Genet Resour Inf 45: 1-14. doi: 10.1017/S1014233909990277

27. Quispe EC. 2010. Estimación del progreso genético de seis esquemas de selección en alpacas (Vicugna pacos L.) Huacaya con tres modelos de evaluación en la región Altoandina de Huancavelica. Tesis de doctorado. Lima: Univ Nacional Agraria La Molina. 144 p.

28. Quispe EC, Poma A, Purroy A. 2013. Características productivas y textiles de la fibra de alpacas de la raza Huacaya. Rev Complut Cien Vet 7(1): 1-29. doi: 10.5209/rev RCCV.2013.v7.n1.41413

29. Rogers $\boldsymbol{G}$ 2006. Biology of the wool follicle: an excursion into a unique tissue interaction system waiting to be rediscovered. Exp Dermatol 15: 931-949. doi: 10.1111/j.1600-0625.2006.00512.x 
30. Safley M. 2005 Crimp versus crinkle. [Internet]. Disponible en: http:// www.alpacas.com/AlpacaLibrary/Html/ CrimpCrinkle.htm

31. Siguayro P. 2009. Comparación de las características físicas de las fibras de la llama Ch'aku (Lama glama) y la alpaca Huacaya (Lama pacos) del Centro Experimental Quimsachata del INIA-Puno. Tesis de Magíster. Lima: Univ Nacional Agraria La Molina. $188 \mathrm{p}$.

32. Software R, V-3.1.2. [Internet]. Disponible en: http://tp.cixug.es/CRAN/

33. Valdivia V. 2009. Relación entre la condición corporal y edad con la calidad de fibra y longitud de mecha del vellón de alpaca Huacaya. Tesis de Médico Veterinario. Lima: Univ Nacional Mayor de San Marcos. 20 p.
34. Wang L, Liu X, Wang X. 2004. Changes in fibre curvature during the processing of wool and alpaca fibres and their blends. En: Chen X, GeY, Yan X (eds). Proc Textile Institute $83^{\text {rd }}$ World Conference. England: The Textile Institute.

35. Wang $X$, Wang L, Liu X. 2003. The quality and processing performance of alpaca fibres. Series RIRDC Publication $N^{\circ} 03 / 128$. Australia: Rural Industries Research and Development Corporation. $118 \mathrm{p}$.

36. Wuliji T, Davis GH, Dodds KG, Turner PR, Andrews RN, Bruce GD. 2000. Production performance, repeatability and heritability estimates for live weight, fleece weight and fiber characteristics of alpacas in New Zealand. Small Rumin Res 37: 189-201. doi: 10.1016/S09214488(00)00127-9 